\title{
LOS ATRIBUTOS DE LOS ESTABLECIMIENTOS EDUCACIONALES QUE PUEDEN PREDECIR LA PREFERENCIA POR PARTE DE LOS APODERADOS
}

\author{
Mario Garay ${ }^{1}$, Mario Sillard ${ }^{2}$
}

\section{RESUMEN}

La presente investigación indaga acerca de cómo se comportó la matrícula con el Sistema de Admisión Escolar (SAE) para el proceso 2020, aplicado por primera vez en todas regiones de Chile. Con ello se busca medir la relación estadística entre ciertos atributos de los establecimientos educacionales, con la cantidad de preferencias que tienen por parte de los apoderados de prekínder, kínder y primero básico. A través de la aplicación de un modelo de regresión lineal múltiple, el conjunto de variables independientes considerados en este estudio explica un tercio de la variabilidad de la demanda que atraen los colegios. El resultado que tienen las escuelas en pruebas estandarizadas (SIMCE) y la proporción de matrícula de estudiantes vulnerables (IVE) se revelan como las principales variables predictoras.

Este estudio busca aportar al debate teórico respecto del fenómeno de elección de establecimiento educacional en Chile y, al mismo tiempo, introducir al SAE y a los datos que genera como fuente de información en el campo de la investigación en educación, lo cual puede ser clave para planificar cómo fortalecer el sistema educativo y entregar una oferta más equilibrada a los(as) estudiantes.

Conceptos clave: Sistema de Admisión Escolar, elección escolar, subvención educativa, Ley de Inclusión, segregación escolar, gestión educativa.

\section{ATTRIBUTES OF EDUCATIONAL INSTITUTIONS THAT MAY PREDICT THE PREFERENCE OF PARENTS AND GUARDIANS}

\section{ABSTRACT}

This research enquires the enrollment behaviour with the School Admission System (SAE) for the period 2020, applied for the first time in all regions of Chile. The study seeks to measure the statistical relationship between certain schools' attributes and the parents' preferences of preschool and first grade students. Through the application of a multiple linear regression model, the set of independent variables considered in this study explains one third of the variability of the demand attracted by schools. The results obtained by schools in standardized testing (SIMCE) and the percentage of vulnerable students enrollment (IVE) appear as the main predictor variables.

This study seeks to contribute to the theoretical debate regarding the phenomenon of choosing an educational institution in Chile. At the same time, it introduces the SAE, and the data that it

1 Universidad de Magallanes, Punta Arenas, Chile. Contacto: mario.garay@umag.cl

2 Universidad de Magallanes, Punta Arenas, Chile. Contacto: mario.sillard@umag.cl 
174 LOS ATRIBUTOS DE LOS ESTABLECIMIENTOS EDUCACIONALES QUE PUEDEN PREDECIR LA PREFERENCIA POR PARTE DE LOS APODERADOS - M. Garay, M. Sillard

generates as a source of information, into the field of educational research, which can be crucial to planning the strengthening of the Educational System and providing a more balanced offer to students.

Key concepts: School Admission System, school choice, educational subsidy, Inclusion Law, school segregation, educational management. 


\section{Presentación del problema}

Con el Sistema de Admisión Escolar (SAE), el proceso de admisión de estudiantes a establecimientos financiados por el Estado deja de ser autogestionado por estas instituciones para, en rigor, terminar con la selección discriminatoria o arbitraria. Quienes se oponen a este nuevo mecanismo lo denominaron "tómbola", pues representa una pérdida de control por parte de estas entidades. No obstante, la incertidumbre declarada por una parte de los apoderados frente a este proceso de postulación de sus hijos no es causada por la centralización del sistema de admisión, sino más bien por la alta concentración de las solicitudes en escuelas "deseables", lo que deja al descubierto la escasez de oferta de calidad percibida en el sistema.

El SAE viene a revitalizar la discusión teórica respecto de la elección de colegios, bien desarrollada en Chile a propósito de la expansión de matrícula escolar de carácter privado (Orellana et al., 2018). Asimismo, el SAE también es capaz de nutrir de información clave a los administradores de la red de educación pública y subvencionada, pues el sistema de admisión estaba íntegramente delegado a los establecimientos.

La presente investigación tiene por objetivo evaluar cómo se comporta la demanda de los establecimientos sobre la base de ciertas características propias. Con principal foco en los niveles de prekínder, kínder y primero básico, se analiza la cantidad preferencias que tiene cada recinto en el proceso SAE 2020 a través de su plataforma web; luego de lo cual se contrastan estas preferencias con una serie de variables independientes que corresponden, principalmente, a indicadores institucionales. Con ello se busca aportar al debate respecto de la elección de establecimiento educacional y a la consideración del SAE como herramienta de gestión educativa.

\section{Antecedentes teóricos}

La revisión de la literatura se divide en tres apartados, configurando el contorno de nuestro objeto de estudio. Primero se hace una revisión de la evolución reciente del sistema educativo en Chile y de la falta 
de asignación de presupuestos para su mejor funcionamiento, lo que generó una crisis de acceso, calidad y segregación educativa en nuestro país, y dio lugar a la Ley No 20.845. En segundo término, pondremos el foco en la evidencia empírica y la literatura referida a la elección de escuela en Chile, y, por último, en los alcances de dicha normativa que genera posteriormente el SAE.

El sistema educativo en Chile contemporáneo. Un proyecto fallido en la reducción de las desigualdades educativas

El sistema escolar chileno está compuesto por tres tipos de establecimientos, según dependencia: municipales (gestionados por municipalidades y servicios locales de educación con financiamiento público), particulares subvencionados (gestionados por organizaciones sin fines de lucro, que perciben subvención estatal por estudiante y que, además, pueden cobrar copago) y privados (gestionados por particulares que no reciben fondos públicos). Con la implementación, en la reforma de 1981, del sistema universal de voucher — que asigna fondos por la cantidad de estudiantes que asiste, independiente de su origen socioeconómico y del tipo de dependencia- se consolida un sistema escolar basado en la libre elección y el subsidio a la demanda, válido para ambos tipos de sostenedores que deben competir por incrementar su matrícula y asistencia (Valenzuela, Labarrera y Rodríguez, 2008). El sentido de esta lógica organizativa apunta a la construcción de un sistema basado en la competencia y fundamentado en el mercado. Este modelo pronostica que las familias preferirían escuelas con buenos resultados académicos y con proyectos educativos afines a sus valores, lo que produce un crecimiento de la educación privada y la consecuente pérdida de matrícula y peso de la educación pública (Bellei, González y Valenzuela, 2010), así como una serie de consecuencias en términos de la calidad, equidad, eficiencia y eficacia del sistema (Villalobos y Quaresma, 2015).

La mayoría de los estudios sobre la educación de nuestro país ha generado un relativo consenso de que las escuelas y liceos en Chile tienen un rol activo en favorecer la segmentación social (Valenzuela, Bellei \& De los Ríos, 2014; Treviño, Valenzuela, 
Villalobos, Béjares, Wyman y Allende, 2018), de modo que el perfil de ingresos económicos de una familia define, en buena parte, el tipo de establecimiento al que asistirán sus hijas e hijos. Algunos especialistas (Peña y Toledo, 2016; Fukushi, 2010; Landerretche y Lillo, 2011) advierten que la consolidación de este modelo atenta contra los valores y principios reconocibles en sociedades como las nuestras, donde las expectativas personales y relatos de cohesión social se direccionan por las ideas de libertad, movilidad social y "meritocracia", las cuales, al no cumplirse materialmente, generan importantes tensiones sociales e inestabilidades matrices del descontento social y político del Chile reciente.

En este sentido, el "corazón" del problema relacionado con la segregación en la educación chilena se relaciona con la forma de financiamiento de las escuelas públicas, pues han sido empujadas a funcionar "como privadas" (Atria, 2012; Bellei, 2015) a partir de la competencia por matrícula y aranceles. La lógica esperada es que, al disputarse los estudiantes (y con ello, el arancel simulado por un subsidio o voucher que trae cada uno al establecimiento), las escuelas mejoren sus prácticas pedagógicas y su gestión para atraer apoderados y captar estudiantes (clientes). Se crea entonces una especie de mercado en el que el pago del arancel está simulado por el financiamiento público "por cabeza".

La administración del sistema educativo en Chile está marcada por la implementación de este procedimiento de voucher y de promoción del "financiamiento compartido", gracias al cual establecimientos privados que reciben recursos del Estado pueden cobrar, simultáneamente, un adicional a los apoderados. Existe evidencia de que ello ha favorecido un proceso de segregación socioeconómica, así como también un problema de sustentabilidad financiera en las escuelas y liceos administrados por municipios. Como consecuencia de esto, la imagen de estos establecimientos se asocia a estudiantes pobres y/o de bajo rendimiento, lo que lleva a los apoderados a evitarlos, provocando un descenso en sus matrículas (Córdoba, 2014) y, por tanto, un círculo vicioso que perjudica la

3 Ley $18.768,1988$. 
oferta educativa para sectores más vulnerables y de bajos ingresos. Si bien la inclusión de actores privados en la oferta educativa, en la historia reciente de Chile, ha colaborado a elevar sustancialmente la cobertura educacional, no resulta del todo claro cómo ha contribuido esto al desempeño pedagógico (Contreras et al., 2011). Lo que es indiscutible es el efecto negativo en la matrícula $-\mathrm{y}$, por tanto, en el financiamiento- de las escuelas del sector público, demostrando la ineficiencia de este sistema en la generación y control de la oferta (Eyzaguirre, Hernando y Blanco, 2018), es decir, el fracaso del modelo de administración basado en la creación de un "mercado educacional" que subsidia la demanda.

Esta situación no solo profundiza la percepción de baja calidad del sistema público por parte de los apoderados, potenciando los efectos adversos sobre el sistema educativo en su globalidad (Orellana, Caviedes, Bellei y Contreras, 2018), sino también la crisis de la segregación educativa en Chile como un efecto derivado de la alta desigualdad de ingresos, que es aún mayor que la segregación residencial (Santos y Elacqua, 2016). En síntesis, estamos frente a una serie de políticas que han favorecido la privatización de un servicio y que, aun aportando significativamente en una ampliación de la cobertura, no han permitido hacer más eficiente el gasto público, ni han proporcionado incentivos suficientes para reducir la elevada influencia de la condición socioeconómica en la calidad del sistema escolar. De este modo, la educación deja de ser un bien universal para ser el privilegio de unos pocos (Programa de las Naciones Unidas para el Desarrollo, 2017).

\section{Elegir/evitar escuela en Chile}

Las políticas de elección de escuela se conciben como programas que brindan a los estudiantes mayores oportunidades de acceso a establecimientos educacionales, más allá de sus áreas de residencia y sus grados de vulnerabilidad. En muchas ocasiones también se planifican como una estrategia que, potencialmente, puede ayudar a reducir los efectos de la segregación residencial y escolar de los niños y jóvenes más desfavorecidos. 
El siguiente elemento por considerar en la configuración de un marco teórico y empírico para efectos de esta investigación, es el fenómeno social tras la elección de escuela por parte de los apoderados. En términos generales, uno de los pilares sustantivos de la lógica del funcionamiento del sistema educativo chileno descansa en el supuesto de "libertad de elección" por parte de las familias al momento de escoger establecimiento (Bellei, 2014). El voucher (subsidio) presupone que el financiamiento del Estado va eficientemente dirigido a las "mejores" instituciones, porque son las preferidas por los apoderados y porque tenderán a querer mejorar sus prácticas y funcionamiento interno para captar más estudiantes. Pero, en la práctica, ha quedado en evidencia que la implementación del voucher universal ha aumentado la estratificación social y socioeconómica de las escuelas, no solo entre las públicas y privadas, sino también entre las particulares subvencionadas (Elacqua, 2012). En este sentido, la literatura destaca el predominio de los efectos negativos de la elección escolar y los mercados educativos sobre la equidad educativa (Musset, 2012; OCDE, 2019), pero varía entre los diferentes sistemas educativos y territorios, para dar lugar a diferentes niveles de segregación.

Mundialmente, en las últimas tres décadas se han ido incorporando mecanismos de mercado a los sistemas educativos otrora eminentemente públicos, masivos y obligatorios-, en los que la elección de escuela pasó de ser algo irrelevante a una conducta de suma importancia para las familias, principalmente en sectores de ingresos medios que intentaron vehiculizar así una estrategia de movilización social ascendente o, al menos, evasiva del descenso. En un meta-análisis en el que se revisan sesenta artículos relacionados con la elección de escuela de distintos países, y las respectivas políticas que la promueven, Orellana, Caviedes, Bellei y Contreras (2016) presentan algunos elementos comunes como:

- los sectores de menores ingresos son los más perjudicados, tanto que en algunos estudios se les configura como "no electores";

- los criterios propiamente académicos no son los relevantes para escoger escuela en sectores de ingresos medios y bajos; 
- las transformaciones recientes en cuanto a volumen y heterogeneidad de los sectores medios en sociedades modernas y su desvinculación paulatina del Estado y las políticas de "bienestar", hacen que elegir escuelas y liceos sea un elemento clave en la configuración de su subjetividad y percepción de protección. En resumen, cuando eligen establecimiento para sus hijos, lo hacen en estrecha relación con un escenario de competencia, segregación y posición de clase (Orellana et al., 2018).

Parte importante de esta literatura cuestiona el concepto "libertad de elección". Desde esta perspectiva, poder escoger establecimiento educacional es, finalmente, una posibilidad que tiene una proporción limitada de apoderados, pues hay "una estrecha relación entre el hecho de poder y saber escoger escuela, con la posesión de recursos económicos y culturales" (Orellana et al., 2018, p. 6) que tenga la familia en relación con la oferta de los colegios, en un sistema que ha potenciado la reproducción social.

Diversas investigaciones sobre las políticas de elección de escuela han ido encontrando conductas y patrones vinculados a este tipo de elección, siempre generando distinciones observables por tramos de ingreso. Por ejemplo, familias de grupos socioeconómicos altos tienden a preferir escuelas en un radio de hasta $4 \mathrm{~km}$, mientras que los grupos de más bajos ingresos consideran un radio de $2,5 \mathrm{~km}$. El efecto salida sigue siendo superior a los movimientos integradores que realizan las familias más vulnerables al escolarizarse con estudiantes más favorecidos (Bonal y Zancajo, 2020). Por ejemplo, el estudio de Bifulco, Ladd y Ross (2009) muestra que las escuelas están más segregadas entre grupos étnicos y clases sociales de lo que lo estarían si todos los estudiantes asistieran a las escuelas más cercanas en términos geográficos. Lo cierto es que, en la mayoría de los sistemas escolares, independientemente del grado de libertad de elección que tienen las familias, la segregación residencial de las ciudades está vinculada con la segregación escolar (Santos y Elacqua, 2016), debido a que "la capacidad/disponibilidad para desplazarse a la escuela rompe el factor condicionante de lo residencial y, por otra parte, exacerba las consecuencias sociales de la fragmentación 
espacial, creando desigualdad en el acceso a los servicios de la ciudad" (Córdoba et al., 2017, p. 11).

Al controlar las restricciones del copago, se demostraba estadísticamente que los apoderados escogen "lo mejor" dentro de lo disponible, considerando su radio de decisión y restricción presupuestaria, mientras los apoderados de bajo nivel educacional (educación básica completa o menor) escogían establecimientos de menor rendimiento y mayor proporción de estudiantes vulnerables, en una especie de "autoexclusión" (Flores y Carrasco, 2014). El nivel socioeconómico influye de manera directa sobre la segregación espacial y es uno de los factores claves para entender sus efectos en un campo de una relativa complejidad conceptual.

Los mecanismos de elección de escuela, por otra parte, están cruzados con un plano sociocultural que se vuelve más relevante en función de los conflictos de segregación socioeconómica previamente descritos. El copago, por ejemplo, no solo afecta la decisión en el sentido de la "elección racional" propiamente educacional, sino que también opera en la lógica de la composición social de la escuela (Hernández y Raczynski, 2015); es decir, facilitaría agrupar a los estudiantes en un mismo establecimiento según sus niveles socioeconómicos y/o habilidades académicas. Esta modalidad de elección de escuela empujaría a los establecimientos a tener mejor gestión académica y curricular para atraer familias y estudiantes acordes a su "perfil".

Otro estudio (Bellei et al., 2016) profundiza en la elección de escuelas entrevistando a familias de comunas de bajos ingresos de la Región Metropolitana. En los principales hallazgos se configura la idea de que en sectores de bajos ingresos las familias "no eligen" (siendo actores pasivos), pero que, al mismo tiempo, existe otro perfil de familia que está en búsqueda intensiva de las mejores opciones posibles en "la parte inferior" del mercado educacional, es decir, buscando las mejores opciones dentro del mundo público o en establecimientos subvencionados con aranceles que estén a su alcance. En este sentido, se puede evidenciar que las familias no se enfrentan al mercado educativo con los mismos recursos económicos 
y culturales; por lo tanto, esta lógica perjudica a las más vulnerables, que ven limitada su elección de escuela (Hernández y Raczynski, 2015; Corvalán y Román, 2016).

Lo anterior indica que buscar mejores opciones educativas no responde a tendencias "arribistas", sino a una percepción de riesgo. Se caracteriza como una disputa por "no bajar" más que por "subir" en la estratificación social, a causa de la elección de escuela (Canales et al., 2016). Es decir, las familias de clase baja ven limitado su campo de actuación y, en muchas ocasiones, aunque existan mecanismos como el SAE, que permite una libre elección, deben "conformarse" con la escuela del barrio, lo que provoca un aumento de la concentración y el aislamiento de los más vulnerables (Kye, 2018).

Esto quiere decir que cierto tipo de escuelas están geográfica o socialmente dispuestas a atender a los estudiantes de sectores de bajos ingresos, lo que les otorga un fuerte estímulo para ser segregadoras y, así, ser competitivas en captar matrícula (y financiamiento). Entonces, la segregación escolar es considerablemente más alta que la segregación residencial, sobre todo en las áreas geográficas que tienen mayores posibilidades de elección de escuelas (Allen, 2007). En especial en aquellas áreas con mayor presencia de escuelas privadas, que muestran un efecto negativo de los márgenes de elección en la segregación escolar, sobre todo en contextos en los que existe una elevada presencia de alternativas de escuelas privadas (Bonal y Zancajo, 2020). Y políticas de libre elección, como el SAE, si bien han aumentado la oferta educativa, también, con ello, han matenido las desigualdades educativas.

\section{La Ley de Inclusión}

Desde el inicio de los años 2000 la clase política dirigente chilena ha recibido una suma de presiones que provienen desde el mundo de la sociedad civil organizada, como también de la evidencia acumulada desde el mundo académico e, inclusive, de organismos internacionales, en cuyos informes destacamos como uno de los países con mayor segregación social en el sistema educativo (PNUD, 2017; Duk y Murillo, 2019). Todas ellas acusan que la disposición del 
sistema educativo, tal como estaba diseñado, agudizaba las diferencias de origen de sus estudiantes y la reproducción de desigualdades, privilegios y exclusiones.

En ese marco, el año 2015, bajo el segundo mandato de la presidenta Michelle Bachelet, emerge el nuevo Sistema de Admisión Escolar (SAE) que, a su vez, es uno de los mecanismos diseñados para implementar la Ley de Inclusión (Ley 20.845). Con ello se dispone que, mediante una plataforma única, se puedan optimizar las preferencias de los apoderados y que, como una medida en pos de la equidad y la justicia, los establecimientos municipales y particulares subvencionados no puedan seleccionar a sus estudiantes. Con la implementación del SAE se desordena, discursivamente, la partición con que operaba la elección de escuelas en el sistema educativo chileno (Canales et al., 2020), y los colegios privados sin subvención del Estado (7\% de la matrícula nacional) quedan excluidos de esta regulación.

Para el funcionamiento del proceso, los apoderados deben ingresar a una plataforma web ${ }^{4}$ y buscar y escoger los establecimientos en una lista ordenada de preferencias. Luego, el sistema procesa de forma simultánea todas las postulaciones y, en caso de haber vacantes disponibles en un establecimiento, son aceptadas. Si existe sobredemanda se aplican criterios de prioridad para postulantes que sean:

- Hermanos de estudiantes ya matriculados en el establecimiento.

- Calificados "prioritarios". El establecimiento debe darle prioridad a un $15 \%$ de estudiantes de familias vulnerables.

- Hijos de funcionarios del establecimiento.

- Exestudiantes del establecimiento que quieran volver (siempre y cuando no hayan sido expulsados).

Luego de considerar esos criterios de prioridad, en caso de haber sobredemanda se aplica la aleatoriedad para asignar las

4 Sistema de Admisión Escolar (SAE). https://www.sistemadeadmisionescolar.cl/comofuncional 
vacantes entre los postulantes, característica más llamativa del SAE con el fin de equiparar probabilidades de acceso. Otrora, los establecimientos que se enfrentaban a esta situación aplicaban filtros, principalmente relacionados con habilidades cognitivas del estudiante o con características socioculturales de los padres, para quedarse con estudiantes con mayor disposición al éxito académico, colaborando sistémicamente a un proceso de segregación y limitando la libertad de elección (Bellei, 2016). Este nuevo escenario busca mejorar las probabilidades de acceso a una educación de calidad para todos los estudiantes, en especial para aquellos niños y jóvenes que no podían acceder a establecimientos municipales de "prestigio" y/o subvencionados que fueran de su preferencia, medidas que, en teoría, buscan reducir desigualdades entre los estudiantes.

En la práctica, la Ley de Inclusión implica ir regulando, progresivamente, el funcionamiento de los establecimientos que reciben recursos públicos, a través de la prohibición de a) copago de los padres, b) selección de estudiantes y c) el lucro. Esto al mismo tiempo que van aumentando los recursos del Estado mediante subvención (voucher), pero con una oferta educativa que posee disímiles atributos de deseabilidad. Entonces, el mayor esfuerzo giraría en torno a la capacidad del sistema para modelar la demanda en un contexto de alta segmentación y a la asignación según los niveles de prestigio percibidos en la oferta (Rodríguez, Espinosa y Padilla, 2020).

Algunos establecimientos privados que recibían recursos del Estado prefirieron renunciar a la subvención (pasándose a la figura de establecimiento privado sin subsidios), para evitar las normativas que regulaban tanto el espacio financiero (retirar utilidades) como el académico (elegir estudiantes). De este modo, se mantienen alejados de sectores sociales estigmatizados (Canales et al., 2016) y conservan sus características de prestigio y capital cultural atribuidos al colegio al momento de ser elegidos por las familias (Berends, 2015).

En específico, y a la luz de las primeras exploraciones investigativas, el SAE resulta ser bastante eficiente en la generación de un nuevo entorno, al rebajar la capacidad de los establecimientos 
para manipular la demanda de admisión a su favor y seleccionar un perfil específico de estudiante. Sin embargo, se aprecia que el nuevo mecanismo aún es deficiente en modificar la distribución de los estudiantes más vulnerables en comparación con el sistema antiguo (Sillard, Garay y Troncoso, 2018).

Como sabemos, el SAE modifica significativamente el proceso de elección y postulación a las escuelas, y "reemplaza un sistema donde las ventajas culturales y sociales tenían un rol fundamental por uno donde existe igualdad efectiva de oportunidades de acceso a toda la oferta escolar financiada por el Estado. Sin embargo, este cambio posee una base de legitimidad moral aún frágil que podría comprometer su sustentabilidad" (Carrasco et al., 2019, p. 7). En este mismo sentido, se indaga a través de 162 entrevistas en profundidad a 81 familiares que pasaron por el SAE en sus primeros años de implementación. Estos relatos manifiestan qué implica este nuevo formato de postulación (a través de una plataforma on line y sin entrevistas o pruebas de selección) y combinan elementos positivos (reducción de tiempos y costos al postular, sensación de equidad y transparencia) con negativos (ansiedad por los resultados o despersonalización del proceso) (Carrasco et al., 2019), información de gran utilidad para ir mejorando el SAE.

Las investigaciones se han ido concentrando en analizar el SAE desde la perspectiva de las familias y su proceso de elección (Duk y Murillo, 2019). Pero otro valor del SAE radica en transparentar la real demanda de los establecimientos públicos y particulares subvencionados, cuestión que anteriormente era invisible, por la autogestión de los colegios en materia de admisión. La implementación del SAE, por tanto, permite hacer visible con precisión, en cada territorio, la cantidad de oferta (vacantes) y de demanda (postulantes), qué instituciones son las más demandadas o visualizadas como de mayor calidad, si las postulaciones están muy concentradas en pocos establecimientos, y otras variantes más. "Esta dimensión de bien público informativo que genera el sistema a partir de las postulaciones no ha sido suficientemente destacada ni explotada" (Eyzaguirre et al., 2018, p. 20). Profundizar en los estudios de las variables del SAE entregaría información útil para una 
mejor comprensión acerca del fenómeno de elección escolar y, a su vez, para alimentar las políticas públicas que permitan abordarlo de manera más efectiva.

Hay poca evidencia empírica aún respecto de cómo impacta el SAE en la interna de los establecimientos educacionales, por ejemplo, en la reconfiguración del tipo de estudiante o de apoderado que ingresa a los colegios. Un estudio desarrollado en Calama (Adones, 2019) describe la transición de un liceo público de alta demanda (que desarrollaba prácticas selectivas) hacia la configuración del primero medio de la generación 2019 mediante el SAE. Tanto docentes como estudiantes encuestados en ese estudio manifestaron resistencia y rechazo al nuevo mecanismo, pues cambiaba el perfil del estudiante que tradicionalmente ingresaba al establecimiento. Lo positivo a favor del SAE, en relación con este estudio, es que satisface un principio de justicia educacional elemental, al conceder a los estudiantes menos aventajados la opción de ser asignados a un colegio de mayor calidad y preferido por su familia (Carrasco y Honey, 2019).

El SAE se ha ido consolidando como un sistema único de admisión, centralizado y transparente, en el que todos los establecimientos compiten bajo las mismas reglas, por lo que la decisión actual giraría en torno a la capacidad para modelar la demanda en un contexto de alta segmentación y con una asignación de atributos percibidos en la oferta que los hacen elegibles.

En este escenario, el objetivo de este artículo es analizar los resultados del proceso de admisión escolar 2020. Focalizándose en los niveles prekínder, kínder y primero básico, se analizan las preferencias que recibe cada establecimiento en el proceso y, luego de ello, se contrastan con una serie de variables predictoras relacionadas con atributos institucionales, geográficos y de vulnerabilidad de las escuelas. Con ello se busca interpretar si hay vínculo estadístico entre dichas variables y la variabilidad de la demanda por parte de los apoderados. 


\section{Metodología}

Esta investigación se realiza en el marco de la postulación a establecimientos educativos financiados por el Estado, a través del Sistema de Admisión Escolar (SAE) del año 2020.

Cabe destacar que el SAE, como mecanismo, lleva cuatro años funcionando. El primer año (2017) se aplicó como marcha blanca solo en la región de Magallanes; el segundo (2018) se implementó adicionalmente en las regiones de Tarapacá, Coquimbo, O'Higgins y Los Lagos. Para 2019 el SAE funcionó en todas las regiones, a excepción de la Metropolitana, que se sumó finalmente en 2020. Durante la aplicación de este último año participaron 526.686 postulantes a 955.220 vacantes, considerando todos los niveles y cursos.

Para ejercicio de esta investigación se extrae la información detallada del SAE 2020, específicamente las vacantes ofrecidas por los establecimientos, la cantidad de preferencias que recibe cada uno y las ubicaciones greográficas de cada institución educativa, como asimismo la de cada uno de los postulantes. Luego se recopilan, desde diversas fuentes públicas ${ }^{5}$, atributos adicionales de las escuelas (ver tabla 5), para enseguida desarrollar el análisis que se propone en los objetivos de este estudio.

El análisis de los datos se realiza a través de un modelo de regresión múltiple lineal de mínimos cuadrados ordinarios (herramienta también conocida como OLS: Ordinal Least Squares), con el propósito de analizar relaciones de dependencia entre los valores de una única variable dependiente y los correspondientes a un número determinado de variables predictoras o independientes.

Por medio de una regresión lineal de este tipo se puede (Cea D’Ancona, 2004):

- Predecir los valores que adoptará una variable dependiente a partir de los valores conocidos de la serie más pequeña posible de

5 Centro de Estudios Mineduc. Datos abiertos. http://datosabiertos.mineduc.cl/ 
variables independientes, es decir, la búsqueda de la ecuación que mejor represente la asociación lineal entre las variables incluidas en el análisis.

- Cuantificar la relación de dependencia (coeficiente de determinación), es decir, calcular la proporción de varianza de la variable dependiente que queda explicada por la conjunción de variables independientes.

- Determinar el grado de confianza con que los investigadores pueden afirmar que la relación observada en los datos muestrales es realmente cierta.

En el gráfico 1 se muestra un resumen del modelo con el que se busca comprender la variabilidad de la cantidad de preferencias que tiene un establecimiento en el SAE 2020.

Variables independientes

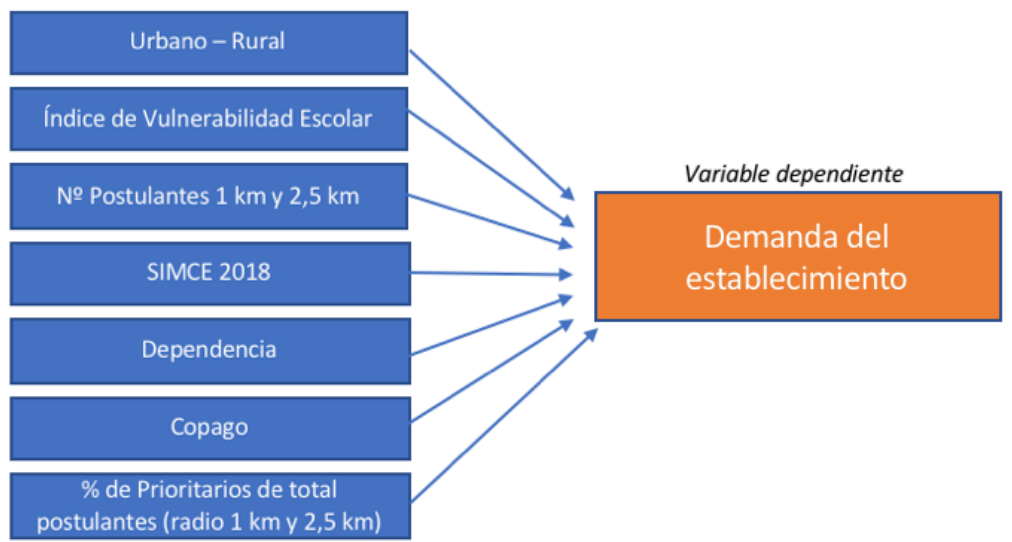

Gráfico 1. Modelo de análisis para regresión múltiple.

La muestra se recoge a partir de una segmentación del SAE 2020 en su proceso de admisión para el mismo año. Consideramos, como elemento basal, que la elección de escuela es un fenómeno social heterogéneo y que depende mucho del nivel en el que se haga. Por ejemplo, una familia que quiere postular a sus hijos a primero medio sigue un proceso muy diferente que el de una familia que postula a su hijo a educación inicial. Por tanto, se buscó poner foco 
en los niveles iniciales en cada establecimiento (prekínder, kínder y primero básico) con los siguientes criterios adicionales:

- Se toman en cuenta solo los establecimientos que tengan cursos a total disposición del proceso SAE 2020. Es decir, que la totalidad de la capacidad de los cursos esté disponible como vacantes. Se considera, por tanto, el primer nivel completamente disponible.

- Para excluir establecimientos que estén muy aislados y, por tanto, tengan muy baja demanda o un comportamiento muy diferente por su situación geográfica, no se considera aquellos que tengan menos postulantes viviendo a 5 kilómetros a la redonda que vacantes.

- Ambos criterios de exclusión/inclusión de establecimiento tienen repercusión en la cantidad de postulantes analizados. Puesto que, como analizamos solo sus primeras preferencias, quedan excluidos aquellos postulantes de prekínder, kínder o primero básico en la medida en que postulen en su primera alternativa a uno de los establecimientos excluidos de la muestra por los puntos anteriores.

- Se toman en cuenta las primeras preferencias de los apoderados solo en fase regular (excluyendo la fase complementaria).

Estos criterios desembocaron en una muestra de 5.266 del total de 7.267 establecimientos (la totalidad que oferta cursos de prekínder, kínder y primero básico).

Con esos criterios, como se puede apreciar en las tablas 1 y 2 , se va configurando la muestra respecto del total de establecimientos y a cada nivel. 
190 LOS ATRIBUTOS DE LOS ESTABLECIMIENTOS EDUCACIONALES QUE PUEDEN PREDECIR LA PREFERENCIA POR PARTE DE LOS APODERADOS - M. Garay, M. Sillard

Tabla 1.

Composición de la muestra comparando con total postulantes y vacantes del SAE 2020

\begin{tabular}{|c|c|c|}
\hline Total Vacantes SAE 2020 & $\begin{array}{l}\text { Vacantes prekínder, } \\
\text { kínder y } 1^{\circ} \text { básico } \\
\text { (Universo del estudio) }\end{array}$ & $\begin{array}{l}\text { Vacantes analizadas en el } \\
\text { estudio } \\
\text { (Muestra del estudio) }\end{array}$ \\
\hline (8.064 establecimientos) & (7.267 establecimientos) & (5.266 establecimientos) \\
\hline \multirow[t]{2}{*}{955.220} & 347.627 & 186.537 \\
\hline & $\begin{array}{l}56 \% \text { del proceso SAE } \\
2020\end{array}$ & $53,6 \%$ del universo \\
\hline $\begin{array}{l}\text { Total postulantes SAE } \\
2020\end{array}$ & $\begin{array}{l}\text { Total postulantes pre- } \\
\text { kínder, kínder y } 1^{\circ} \text { básico } \\
\text { (Universo del estudio) }\end{array}$ & $\begin{array}{l}\text { Postulantes considerados } \\
\text { en la muestra: } \\
\text { (Muestra del estudio) }\end{array}$ \\
\hline \multirow[t]{2}{*}{526.686} & 294.006 & 242.591 \\
\hline & $\begin{array}{l}36 \% \text { del proceso SAE } \\
2020\end{array}$ & $82,5 \%$ del universo \\
\hline
\end{tabular}

Fuente: Elaboración propia con base en datos del MINEDUC.

En la tabla 1 se puede apreciar que más de la mitad (56\%) de las personas que participa en un proceso regulado por el SAE 2020 lo hace para ingresar a los tres primeros niveles de educación inicial. A su vez, en el mismo proceso, un 36\% de las vacantes dispuestas son de dichos niveles.

En la primera parte de la tabla 1 están descritas las vacantes en las tres dimensiones: total de vacantes del proceso; luego, solo las vacantes para los niveles analizados en el estudio (prekínder, kínder y primero básico) y, por último, las vacantes consideradas en la muestra. Del mismo modo, abajo, en la tabla 1, están los datos para los postulantes. Este proceso de segmentación de cómo se fue configurando la muestra queda representado en el gráfico 2. Como se puede apreciar, el presente estudio delimita como muestra al 30\% de las vacantes y al $46 \%$ de los postulantes de la totalidad del proceso SAE 2020. 
Tabla 2.

Resumen: vacantes y postulantes por nivel, SAE 2020

\begin{tabular}{lrrrr}
\hline & \multicolumn{2}{c}{ Totalidad del proceso } & \multicolumn{2}{c}{ Muestra } \\
\hline & Vacantes & Postulantes & Vacantes & Postulantes \\
Prekínder & 166.237 & 155.320 & 152.710 & 129.333 \\
Kínder & 73.825 & 63.441 & 13.128 & 51.666 \\
$1^{\circ}$ Básico & 117.565 & 75.245 & 20.699 & 61.592 \\
Totales & 357.627 & 294.006 & 186.537 & 242.591 \\
\hline
\end{tabular}

Fuente: Elaboración propia con base en datos del MINEDUC.

En la tabla 2 se detalla la cantidad de vacantes y postulantes por nivel. La diferencia entre la totalidad del proceso y la de la muestra, tal como se explicó, es porque solo se consideran los establecimientos que ofrecen el "curso vacío" (cupos igual a vacantes) al proceso SAE 2020. De esta manera, las escuelas pueden compararse entre sí en relación a cuántas preferencias tienen en el proceso y qué establecimientos tienen baja demanda o sobredemanda.

De la tabla 2 se desprende que, globalmente, en cada nivel, hay más vacantes que postulantes, lo que pudiera hacer presumir, a priori, que hay opciones para que todos los postulantes tengan acceso a un cupo. Sin embargo, no todas las vacantes se distribuyen geográficamente de forma relacionada con la densidad demográfica de los postulantes. Por un lado, hay una sobreoferta en lugares apartados o rurales, y, al mismo tiempo, existe sobredemanda en sectores más urbanizados y residenciales. Esto se puede apreciar cuando hacemos la muestra para el estudio. Como nos quedamos solamente con aquellas escuelas que tienen (como mínimo) igual número de vacantes que de postulantes viviendo en un radio de 5 kilómetros, vemos que en ese subconjunto de los establecimientos educacionales la relación vacante/postulante ya no es holgada, sino incluso preocupante.

En síntesis, si solo tomamos en cuenta los 5.266 (y no el total, 7.267) establecimientos ubicados en donde hay igual o más postulantes que vacantes viviendo a $5 \mathrm{~km}$, tenemos solo $186 \mathrm{mil}$ vacantes para más de 242 mil postulantes. Esto implica que el SAE genera un entorno favorable para comprender la sobreoferta de escuelas o la sobredemanda de postulantes en distintas zonas 
de Chile. De este modo, se puede obtener una estimación de la "capacidad ociosa" de los establecimientos que no son demandados por las familias (Eyzaguirre et al., 2018).

En la tabla 3 se vinculan algunas características del establecimiento en relación con la cantidad de sus "primeras preferencias" en la operacionalización de las variables. Por ejemplo, del total de vacantes de la muestra, un $80 \%$ de ellas son de establecimientos sin copago. Sin embargo, un 33\% de los postulantes prefiere establecimiento con copago como primera preferencia. Sucede lo mismo con los establecimientos particulares subvencionados: ofrecen un $61 \%$ de las vacantes, pero reciben un $76 \%$ de las primeras preferencias de los postulantes.

Tabla 3.

Establecimientos, vacantes y postulantes por urbano-rural, copago y dependencia

\begin{tabular}{|c|c|c|c|c|c|}
\hline \multirow[b]{2}{*}{ Urbano } & \multirow{2}{*}{$\begin{array}{c}\text { Establecimientos } \\
3.937\end{array}$} & \multicolumn{2}{|c|}{ Vacantes } & \multicolumn{2}{|c|}{$\begin{array}{c}\text { Postulantes que } \\
\text { ponen como primera } \\
\text { preferencia: }\end{array}$} \\
\hline & & 169.523 & $91 \%$ & 230.638 & $95 \%$ \\
\hline Rural & 1.329 & 17.014 & $9 \%$ & 11.953 & $5 \%$ \\
\hline Sin copago & 4.551 & 148.487 & $80 \%$ & 161.836 & $67 \%$ \\
\hline Con copago & 715 & 38.050 & $20 \%$ & 80.755 & $33 \%$ \\
\hline $\begin{array}{l}\text { Municipal o Serv. Local } \\
\text { Part. Subvencionado }\end{array}$ & $\begin{array}{l}2.657 \\
2.609\end{array}$ & $\begin{array}{r}72.951 \\
113.586\end{array}$ & $\begin{array}{l}39 \% \\
61 \%\end{array}$ & $\begin{array}{r}60.277 \\
182.314\end{array}$ & $\begin{array}{l}25 \% \\
75 \%\end{array}$ \\
\hline
\end{tabular}

Fuente: Elaboración propia con base en datos del MINEDUC

Elemento clave, en términos metodológicos, es cómo se configura la variable dependiente.

Como se puede apreciar en la tabla 4, tenemos tres formas de operacionalizar la variable dependiente. Un mecanismo inicial es el simple conteo de primeras preferencias que tiene un establecimiento. Luego, una segunda forma considera el tamaño de un establecimiento y la demanda que recibe de manera simultánea, para lo cual se calcula el cociente entre preferencias y vacantes. De esta forma, si 
un establecimiento tiene igual cantidad de vacantes que de primeras preferencias, tendrá en esta variable un resultado igual a 1. En tanto, si un establecimiento tiene valores mayores a 1 , tiene más preferencias que vacantes, y si tiene valores menores a 1 , menos preferencias que vacantes. En nuestra muestra, cabe destacar, solo un 37,5\% tiene más primeras preferencias que vacantes.

Con ambas formas de medir la demanda de un establecimiento, esta queda distribuida de manera "anormal", con muchos valores aislados y con amplios niveles de dispersión, lo cual perjudica el análisis posterior de la regresión múltiple al no haber normalidad en la distribución de su variable dependiente. Para resolver eso, las Preferencias/Vacantes se llevan a una escala logarítmica, tal como recomienda Cea D'Ancona (2004) cuando la "variable dependiente muestra asimetría positiva severa".

Tabla 4.

Medidas para primeras preferencias, preferencias/vacantes y preferencias/vacantes (log)

\begin{tabular}{lccc}
\hline & $\begin{array}{c}\text { Total primeras } \\
\text { preferencias }\end{array}$ & $\begin{array}{c}\text { Preferencias/ } \\
\text { Vacantes }\end{array}$ & $\begin{array}{c}\text { Preferencias/ } \\
\text { Vacantes (Log) }\end{array}$ \\
\cline { 2 - 4 } Media & 46,07 & 1,07 & $-0,11$ \\
Mediana & 21,5 & 0,73 & $-0,1$ \\
Desv. típ. & 70,58 & 1,22 & 0,41 \\
Mínimo & 0 & 0 & $-1,65$ \\
Máximo & 1,178 & 16,83 & 1,23 \\
Z de Kolmogorov-Smirnov & 18,648 & 13,688 & 2,218 \\
Sig. asintót. (bilateral) & $\mathrm{p}<0,00001$ & $\mathrm{p}<0,00001$ & $\mathrm{p}=0,0001$ \\
\hline
\end{tabular}

Fuente: Elaboración propia.
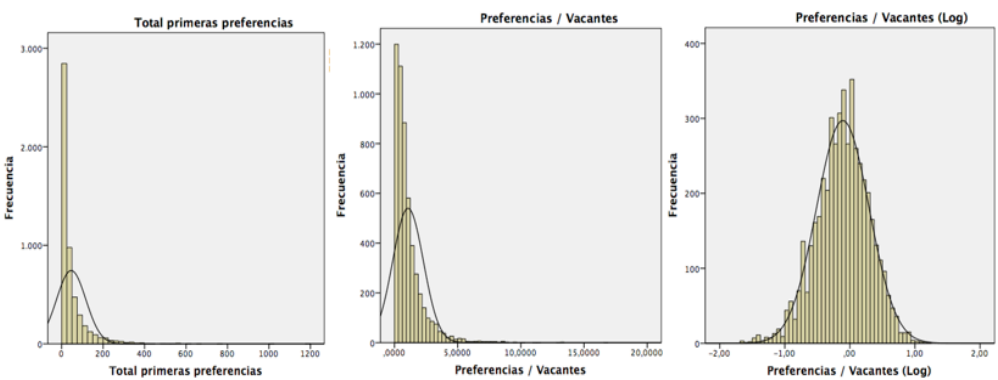

Gráfico 2. Histogramas de distribución para total de primeras preferencias, preferencias/ vacantes y preferencias/vacantes (log).

Fuente: Elaboración propia. 
En la tabla 4 podemos comparar los distintos resultados estadísticos de las variables en los que se incluye el Test de Normalidad de Kolmogorov-Smirnov. Si bien el valor de significancia es cercano a 0 (se rechaza la hipótesis nula de que la distribución es normal), se puede apreciar que la distribución se va "normalizando" y que la escala logarítmica presenta el mejor cálculo de valor $\mathrm{Z}$ y valor P. Ello, además, lo podemos ir visualizando con los histogramas del gráfico 2 .

Respecto de las variables independientes, las podemos agrupar en tres grupos:

- Primero, las características básicas de la institución. Aquí consideramos las relacionadas con su dependencia administrava (privada subvencionada o pública), si tiene copago (pago arancel por parte de los apoderados) y si está en un sector urbano o rural.

- Segundo, las relacionadas con indicadores institucionales vinculados a mecanismos de rendición de cuentas. Se agrupan aquí:

- Los puntajes de la prueba SIMCE (Sistema de Medición de Calidad en la Educación), producto de un test de Lenguaje y uno de Matemática a los estudiantes de manera censal (a todos los establecimientos del país) y cuyos resultados son públicos.

- Los puntajes IVE (Índice de Vulnerabilidad Escolar), indicador medido por la Junta Nacional de Auxilio Escolar y Becas (JUNAEB) con el objetivo de caracterizar las condiciones socieconómicas de sus estudiantes. De esta manera, cada establecimiento financiado por el Estado declara públicamente la proporción de sus estudiantes matriculados en condición de vulnerabilidad.

- Los indicadores de Desarrollo Personal y Social (IDPS) 6 que se generan a través de la aplicación de cuestionarios denominados "Calidad y Contexto de la Educación", al

6 Para este estudio se consideran los siguientes IDPS: Convivencia y Clima Escolar, Autoestima Académica, Convivencia y Clima Escolar, Hábitos de Vida Saludable y Formación Ciudadana. 
mismo tiempo que se están aplicando los cuestionarios de las pruebas SIMCE ${ }^{7}$.

- En tercer lugar están las variables relacionadas con la ubicación geográfica del establecimiento en relación con los domicilios de los postulantes ${ }^{8}$. Del total de postulantes de nuestra muestra, solo un 16\% de ellos participó del proceso SAE 2020 con una ubicación no computable por el sistema de geolocalización; por tanto, quedaron excluidos de esta medición. Luego, a partir del mapeo de los postulantes se generan dos variables:

- La cantidad de postulantes cercanos al establecimiento en un radio de 1 y 2,5 kilómetros.

- La proporción de familias vulnerables (prioritarias) postulantes que hay en un radio de 1 y 2,5 kilómetros en relación con la totalidad de familias que postularon.

En la tabla 5 se presentan las variables de estudio y su respectiva operacionalización. En primer lugar, la variable dependiente y, luego, en la lista, le siguen las demás variables independientes consideradas en el estudio.

7 Completo detalle respecto de la construcción de estos indicadores, sus componentes y su validación, en el Informe Técnico 2017, Indicadores de Desarrollo Persona y Social (IDPS), descargable en: https://www.agenciaeducacion.cl/evaluaciones/indicadores-desarrollopersonal-social/

8 Este cálculo se realizó con el software RStudio (entorno de desarrollo integrado para el lenguaje de programación R), junto con los "paquetes" denominados "sf" y "leaflet", para delimitar los puntos GPS asociados a cada establecimiento y a cada postulante, en los radios de distancia definidos para esta investigación. 
Tabla 5.

Operacionalización de variables de estudio

\begin{tabular}{|c|c|c|}
\hline Variable & Descripción & Operacionalización \\
\hline $\begin{array}{l}\text { Demanda del } \\
\text { establecimiento }\end{array}$ & $\begin{array}{l}\text { Cantidad de primeras preferencias que } \\
\text { tiene un establecimiento en el SAE } \\
\text { Controlada por cantidad de vacantes y } \\
\text { llevada a escala logarítmica }\end{array}$ & Cuantitativa \\
\hline Urbano - Rural & $\begin{array}{l}\text { Ubicación escuela según Directorio } \\
\text { MINEDUC }\end{array}$ & $0=$ Urbano, $1=$ Rural \\
\hline IVE & $\begin{array}{l}\text { Índice de Vulnerabilidad Escolar, } \\
\text { medido por JUNAEB. Entrega indicador } \\
\text { respecto de la proporción de matrícula en } \\
\text { situación de pobreza }\end{array}$ & Cuantitativa (\%) \\
\hline $\begin{array}{l}\text { Postulantes } 1 \mathrm{~km} \\
\text { y } 2,5 \mathrm{~km}\end{array}$ & $\begin{array}{l}\text { Cantidad de postulantes proceso } \\
2020 \text { viviendo a } 1 \mathrm{~km} \text { y } 2,5 \mathrm{~km} \text { del } \\
\text { establecimiento }\end{array}$ & Cuantitativa \\
\hline SIMCE Lenguaje & $\begin{array}{l}\text { Puntaje SIMCE Lenguaje, } 4^{\circ} \text { básico año } \\
2018\end{array}$ & Cuantitativa \\
\hline $\begin{array}{l}\text { SIMCE } \\
\text { Matemática }\end{array}$ & $\begin{array}{l}\text { Puntaje SIMCE Matemática, 4º básico año } \\
2018\end{array}$ & Cuantitativa \\
\hline $\begin{array}{l}\text { Autoestima } \\
\text { académica }\end{array}$ & $\begin{array}{l}\text { Indicador de Desarrollo Personal y Social, } \\
\text { evaluado por la Agencia de Calidad }\end{array}$ & $\begin{array}{l}\text { Cuantitativa (Puntaje } \\
\text { de } 1 \text { a 100) }\end{array}$ \\
\hline $\begin{array}{l}\text { Convivencia y } \\
\text { clima escolar }\end{array}$ & $\begin{array}{l}\text { Indicador de Desarrollo Personal y Social, } \\
\text { evaluado por la Agencia de Calidad }\end{array}$ & $\begin{array}{l}\text { Cuantitativa (Puntaje } \\
\text { de } 1 \text { a 100) }\end{array}$ \\
\hline $\begin{array}{l}\text { Hábitos vida } \\
\text { saludable }\end{array}$ & $\begin{array}{l}\text { Indicador de Desarrollo Personal y Social, } \\
\text { evaluado por la Agencia de Calidad }\end{array}$ & $\begin{array}{l}\text { Cuantitativa (Puntaje } \\
\text { de } 1 \text { a 100) }\end{array}$ \\
\hline $\begin{array}{l}\text { Formación } \\
\text { ciudadana }\end{array}$ & $\begin{array}{l}\text { Indicador de Desarrollo Personal y Social, } \\
\text { evaluado por la Agencia de Calidad }\end{array}$ & $\begin{array}{l}\text { Cuantitativa (Puntaje } \\
\text { de } 1 \text { a 100) }\end{array}$ \\
\hline Dependencia & $\begin{array}{l}\text { Tipo de administración del } \\
\text { establecimiento educacional }\end{array}$ & $\begin{array}{l}\text { Dicotómica. } 0= \\
\text { Público, } 1 \text { = Particular } \\
\text { Subvencionado }\end{array}$ \\
\hline Copago & $\begin{array}{l}\text { El establecimiento exige pago de arancel o } \\
\text { matrícula a los apoderados }\end{array}$ & $\begin{array}{l}\text { Dicotómica. } 0=\text { Sin } \\
\text { Copago, } 1=\text { Con } \\
\text { Copago }\end{array}$ \\
\hline $\begin{array}{l}\% \text { de Postulantes } \\
\text { Prioritarios } \\
\text { (radio } 1 \mathrm{~km} \mathrm{y} \\
2,5 \mathrm{~km} \text { ) }\end{array}$ & $\begin{array}{l}\text { Proporción de postulantes "prioritarios" } \\
\text { con base en el total de postulantes totales } \\
\text { que viven en un radio a } 1 \text { y } 2,5 \mathrm{~km} \text { de la } \\
\text { escuela }\end{array}$ & Cuantitativa (\%) \\
\hline
\end{tabular}

Fuente: Elaboración propia.

\section{Análisis de datos}

Se aplicó un análisis de regresión múltiple, en el que se tomó como variable dependiente la cantidad de demanda que tenía cada establecimiento, calculando el nivel de vínculo que tienen las variables independientes y cuánto podría explicar nuestro modelo. 
Tabla 6 .

Coeficientes de correlación entre variables independientes y variable dependiente

\begin{tabular}{llcc}
\hline Curalidad & $\begin{array}{c}\text { Demanda del } \\
\text { establecimiento }\end{array}$ & Sig. \\
\hline X1 & (r) & \\
X2 & Copago & $-0,206$ & $\mathrm{p}<0,01$ \\
X3 & Dependencia & 0,338 & $\mathrm{p}<0,01$ \\
X4 & SIMCE Lenguaje & 0,314 & $\mathrm{p}<0,01$ \\
X5 & SIMCE Matemática & 0,336 & $\mathrm{p}<0,01$ \\
X6 & IVE & 0,357 & $\mathrm{p}<0,01$ \\
X7 & Cantidad de postulantes cerca $(1 \mathrm{~km})$ & $-0,425$ & $\mathrm{p}<0,01$ \\
X8 & Cantidad de postulantes cerca $(2,5 \mathrm{~km})$ & 0,197 & $\mathrm{p}<0,01$ \\
X9 & \% de Prioritarios $(1 \mathrm{~km})$ & 0,138 & $\mathrm{p}<0,01$ \\
X10 & \% de Prioritarios (2,5 km) & $-0,156$ & $\mathrm{p}<0,01$ \\
X11 & Autoestima académica & $-0,148$ & $\mathrm{p}<0,01$ \\
X12 & Convivencia y clima escolar & 0,077 & $\mathrm{p}<0,01$ \\
X13 & Hábitos vida saludable & 0,110 & $\mathrm{p}<0,01$ \\
X14 & Formación ciudadana & 0,167 & $\mathrm{p}<0,01$ \\
\hline
\end{tabular}

Fuente: Elaboración propia.

Como se puede apreciar en la tabla 7, el Índice de Vulnerabilidad Escolar (IVE) aporta la correlación más importante, que ocurre de forma inversa: a medida que aumenta la proporción de estudiantes vulnerables matriculados en una escuela, baja la cantidad de demanda que tiene el establecimiento. Luego vienen los puntajes SIMCE (Matemática, $r=, 357$ y Lenguaje, $r=336$ ), junto con copago (,338), con puntajes de correlación más intensos.

Otro elemento a destacar es que mientras más estudiantes vivan cerca de la escuela, más demanda tendrá ésta (variables X7 y X8). Esa correlación no es tan intensa (radio de $1 \mathrm{~km}, \mathrm{r}=, 197$ y radio de $2,5 \mathrm{~km}, \mathrm{r}=, 138$ ), pero lo relevante es cómo, al mismo tiempo, la proporción de estudiantes prioritarios viviendo cerca respecto del total de postulantes genera una correlación similar, pero inversa (X9 y X10).

De esta manera, se aplicó un procedimiento de inclusión de cada variable en el formato "paso a paso". El programa SPSS va incorporando las variables independientes una a una, en la medida en que aumente el nivel explicativo de forma estadísticamente 
significativa del modelo, sin aumentar la colinealidad. Para ello, el valor de "F de entrada" es de 2,07, y de 1,07 el "de salida", tal como lo recomienda Afifi y Clark (1990, citado en Cea D’Acona, 2004).

Considerando estos criterios, al aplicar la regresión lineal múltiple es posible obtener los resultados que pueden verse en las tablas 8 y 9 . El modelo excluye a las variables, ya sea por poca significatividad estadística (como es el caso de UrbanoRural, Formación Ciudadana, Convivencia y Clima Escolar) o por colinealidad con otras variables que explican mejor la variable dependiente, tal como es el caso de Copago (cuya correlación queda mejor explicada por Dependencia) y SIMCE Matemática (que es superado por SIMCE Lenguaje).

Tabla 7.

Coeficientes de regresión múltiple

\begin{tabular}{|c|c|c|c|c|c|c|c|}
\hline & \multicolumn{2}{|c|}{$\begin{array}{l}\text { Coeficientes no } \\
\text { estandarizados }\end{array}$} & \multicolumn{3}{|c|}{$\begin{array}{l}\text { Coeficientes } \\
\text { tipificados }\end{array}$} & \multicolumn{2}{|c|}{$\begin{array}{c}\text { Estadísticos de } \\
\text { colinealidad }\end{array}$} \\
\hline & $\mathrm{B}$ & Error típ. & Beta & $\mathrm{t}$ & Sig. & Tolerancia & FIV \\
\hline Intercepto & 0,057 & 0,098 & & 0,587 & 0,558 & & \\
\hline IVE 2019 & $-1,316$ & 0,078 & $-0,31$ & $-16,852$ & $\mathrm{p}<0,01$ & 0,455 & 2,198 \\
\hline SIMCE Mat & 0,003 & $<0,001$ & 0,245 & 17,328 & $\mathrm{p}<0,01$ & 0,768 & 1,302 \\
\hline Dependencia & 0,113 & 0,011 & 0,141 & 9,976 & $\mathrm{p}<0,01$ & 0,769 & 1,301 \\
\hline $\begin{array}{l}\text { Hábitos vida } \\
\text { saludable }\end{array}$ & 0,009 & 0,001 & 0,166 & 8,931 & $p<0,01$ & 0,447 & 2,236 \\
\hline $\begin{array}{l}\text { Autoestima } \\
\text { académica }\end{array}$ & $-0,008$ & 0,001 & $-0,116$ & $-6,171$ & $\mathrm{p}<0,01$ & 0,439 & 2,277 \\
\hline $\begin{array}{l}\% \text { de } \\
\text { prioritarios } \\
(2,5 \mathrm{~km})\end{array}$ & 0,16 & 0,042 & 0,06 & 3,794 & $\mathrm{p}<0,01$ & 0,61 & 1,638 \\
\hline $\begin{array}{l}\text { Postulantes } \\
\text { cerca (1 km) }\end{array}$ & \multicolumn{2}{|c|}{$9,15 \mathrm{E}-05<0,001$} & 0,119 & 6,042 & $\mathrm{p}<0,01$ & 0,395 & 2,531 \\
\hline Copago & 0,027 & 0,018 & 0,025 & 1,509 & 0,131 & 0,575 & 1,739 \\
\hline
\end{tabular}

Variable dependiente: Preferencias / Vacantes (Log)

Fuente: Elaboración propia.

En la tabla 7 se encuentran los datos de la regresión múltiple y, también, los indicadores de colinealidad de las variables que fueron quedando en el modelo óptimo. En los coeficientes tipificados se puede apreciar los valores de "aporte" de cada variable a la pendiente de la recta que explica la cantidad de preferencias que tendrá un establecimiento. Como se puede observar, las variables de SIMCE y 
de IVE son las dos primeras variables que más incidencia tienen o mejor predicen la cantidad de primeras preferencias.

Es necesario destacar las últimas dos variables incorporadas al modelo. La cantidad de postulantes cerca de la escuela y la proporción de postulantes vulnerables cerca de la escuela tienen un comportamiento estadístico llamativo. Es importante señalar que la densidad demográfica (medible por estas dos variables) queda por debajo en predecir la demanda de una escuela después de indicadores institucionales como el SIMCE y el IVE. Es decir, para esta medición, una escuela no tendría más demanda por estar en un lugar geográfico favorable (más cerca de los postulantes), al menos en la captación de demanda por parte del establecimiento. Adicionalmente, en particular la variable vinculada a la proporción de estudiantes vulnerables (X9 y X10) que viven cerca del establecimiento está incluida en el modelo óptimo de regresión, pero con baja incidencia estadística. Esto ocurre en su interacción con el IVE: la vulnerabilidad escolar ya matriculada en una escuela es mejor predictora que X9 y X10, tanto así que cambia la direccionalidad de la correlación. Nótese en la tabla 7 que ambas tienen correlación inversa: más proporción de estudiantes vulnerables cerca de la escuela, menor cantidad de preferencias. Sin embargo, cuando se hace la regresión múltiple, la variable IVE explica mejor la relación inversa relacionada con la vulnerabilidad y, finalmente, X10 queda como aporte "positivo". 
Tabla 8.

Resumen del modelo de regresión múltiple: variable dependiente: demanda del establecimiento

\begin{tabular}{|c|c|c|c|c|c|}
\hline \multicolumn{6}{|c|}{ Resumen del modelo (a) } \\
\hline Modelo & $\mathrm{R}$ & R cuadrado & $\begin{array}{l}\text { R cuadrado } \\
\text { corregida }\end{array}$ & $\begin{array}{l}\text { Error típ. de } \\
\text { la estimación }\end{array}$ & Durbin-Watson \\
\hline 1 & 0,426 & 0,181 & 0,181 & 0,363 & \\
\hline 2 & 0,503 & 0,253 & 0,253 & 0,346 & \\
\hline 3 & 0,524 & 0,274 & 0,274 & 0,341 & \\
\hline 4 & 0,532 & 0,283 & 0,282 & 0,339 & \\
\hline 5 & 0,538 & 0,290 & 0,289 & 0,332 & \\
\hline 6 & 0,542 & 0,293 & 0,293 & 0,337 & \\
\hline 7 & 0,543 & 0,295 & 0,294 & 0,337 & \\
\hline 8 & 0,548 & 0,300 & 0,299 & 0,335 & 1,861 \\
\hline \multicolumn{6}{|c|}{ 1. Variables predictoras: (Constante), X6 } \\
\hline \multicolumn{6}{|c|}{ 2. Variables predictoras: (Constante), X6, X5 } \\
\hline \multicolumn{6}{|c|}{ 3. Variables predictoras: (Constante), X6, X5, X3 } \\
\hline \multicolumn{6}{|c|}{ 4. Variables predictoras: (Constante), X6, X5, X3, X13 } \\
\hline \multicolumn{6}{|c|}{ 5. Variables predictoras: (Constante), X6, X5, X3, X13, X11 } \\
\hline \multicolumn{6}{|c|}{ 6. Variables predictoras: (Constante), X6, X5, X3, X13, X11, X10 } \\
\hline \multicolumn{6}{|c|}{ 7. Variables predictoras: (Constante), X6, X5, X3, X13, X11, X10, X7 } \\
\hline \multicolumn{6}{|c|}{ 8. Variables predictoras: (Constante), X6, X5, X3, X13, X11, X10, X7, X2 } \\
\hline a Variable & dependi & : Preferencias & acantes (Log) & & \\
\hline
\end{tabular}

Fuente: Elaboración propia.

En la tabla 9, finalmente (que se incluye en anexo), se puede ver el resumen del Modelo, en el que se puede apreciar qué variables se fueron incluyendo hasta lograr un $\mathrm{R}^{2}$ de 0,30 . Es decir, el conjunto de variables predictoras en el Modelo 8 es capaz de explicar un 30\% de la varianza de la demanda de un establecimiento.

\section{Conclusiones}

Los datos proporcionados por el SAE, a partir de su implementación, son de gran utilidad para que el sistema educacional determine la relación oferta-demanda, la capacidad ociosa del sistema y cómo se comportan las preferencias de las familias, lo cual es información relevante para comprender mejor los fenómenos asociados a la elección de escuela y la segregación escolar.

La investigación revela cómo la vulnerabilidad, que se evalúa a través del IVE, afecta las preferencias de los establecimientos. Dicha variable, por sí sola, en una regresión lineal logra un $\mathrm{R}^{2}$ igual a 0,18. 
Cabe destacar que la interacción es inversa, es decir, por cada aumento de una desviación estándar del IVE de un establecimiento, la escuela obtiene -0,425 desviaciones estándar de la cantidad de demanda. El peso de IVE luego, en la interacción con otras variables, se dispersa en el modelo óptimo de regresión múltiple, bajando su coeficiente beta a -0,310. Si bien el SAE busca que los establecimientos no tengan la capacidad de filtrar a sus estudiantes, el estudio muestra que aquellos con mayor IVE tendrían menor demanda. Probablemente se debe a un comportamiento de las familias de evitar establecimientos con alta concentración de estudiantes vulnerables, lo cual persiste con el SAE y no estaría generando un reordenamiento de la matrícula a favor de optimizar gradualmente la recomposición social de las escuelas (Sillard, Garay y Troncoso, 2018). Esto se explica, en parte, debido a la implementación gradual del fin al copago que, aún presente en algunos establecimientos, sigue siendo un mecanismo de exclusión para las familias de nivel socioeconómico bajo, además de ser una política incompatible con la libertad de elección de los padres y/o apoderados.

A la luz de los datos obtenidos, la proporción de matrícula vulnerable de un año en una escuela sigue prediciendo la cantidad de demanda del año siguiente. Por consiguiente, la elección de escuela está condicionada en gran medida por los recursos económicos que definen el rango de opciones que nos ofrece el mercado educativo.

El factor demográfico (o bien, de densidad poblacional) está considerado a través de dos variables independientes: la cantidad de postulantes viviendo en un radio de 1 y 2,5 kilómetros, y la proporción de postulantes de familias prioritarias respecto del total de postulantes en idénticos radios. Si bien estas variables presentan niveles estadísticamente significativos en explicar la varianza, el peso es menor comparado con otros grupos de variables (como las relacionados con la vulnerabilidad o la rendición de cuentas). Esto es coincidente con la evidencia reciente, que indica que una parte importante de estudiantes, en contextos urbanos, opta por no asistir a escuelas cercanas, y que hacen grandes desplazamientos para asistir a un establecimiento escolar en búsqueda de mejores alternativas, según su percepción (Córdoba, Farris y Rojas, 2017). No obstante, 
en un estudio como este, que considera a todos los establecimientos del país, es preciso tomar en cuenta algunos posibles sesgos: por ejemplo, las ciudades tienen distinta densidad demográfica y, eventualmente, un establecimiento ubicado en una zona con alta densidad poblacional puede verse afectado, porque también hay una alta oferta de establecimientos.

Sin embargo, por otra parte, los análisis en esta materia, en futuras investigaciones y mediciones, podrían ampliarse y diversificarse. Es posible que, si recortamos el análisis a regiones específicas de Chile, o si volvemos a hacer el análisis excluyendo escuelas muy aisladas o muy demandadas en ciertas comunas, pueda encontrarse en lo demográfico alguna tendencia más marcada. Con los datos proporcionados por el SAE podría medirse qué porcentaje de los postulantes de una escuela son "vecinos", identificar qué eligen las familias si están a similar distancia de dos o más escuelas, o qué distancias promedio están dispuestos a recorrer en su primera preferencia.

Cabe destacar, además, que la distancia al establecimiento fue considerada de distintas formas (incorporándola en forma cuadrática, para estimar si existía relación no lineal) y no se encontró un vínculo estadístico más fuerte. Al mismo tiempo, se hizo un análisis por separado, considerando establecimientos solo con bajo y alto SIMCE, y la cantidad de postulantes cerca del establecimiento tampoco subió en su incidencia.

La importancia que tienen las variables institucionales (comúnmente relacionadas con la rendición de cuentas) es signifiticativa. Los puntajes SIMCE tienen una considerable correlación con la cantidad de preferencias que recibe un establecimiento.

Haciendo el ejercicio de aislar la regresión que existe entre SIMCE Matemática y la cantidad de preferencias, encontramos un valor $R^{2}=0,127$. Lo que implica una variación de 0,439 desviaciones estándar de las preferencias por vacante al subir una desviación estándar de SIMCE. 
Lo anterior se debe a un sistema educativo altamente segregado, en el que el prestigio de los colegios se mide bajo un criterio de competencia por la matrícula de los estudiantes. En este sentido, el SIMCE provee de información respecto de la calidad de las escuelas y busca que las familias no escojan a los establecimientos con bajos rendimientos. Si bien el SAE abre sus puertas a nuevas ofertas educativas, las familias de clase baja ven limitado su radio de acción, prefieren menor calidad y colegios menos sobredemandados (Carrasco y Honey, 2018), lo que no genera cambios significativos en la brecha del acceso a establecimiento de "calidad".

Esto implica un hallazgo interesante desde el punto de vista de la investigación educativa, en cuanto se pueda cuantificar concretamente la incidencia estadística en la demanda y preferencia por parte de los apoderados para escoger escuelas que tienen principalmente las pruebas estandarizadas como el SIMCE. Lo anterior, entendiendo por cierto que la comprobación de estas hipótesis se hace dentro de los límites metodológicos que conlleva y que, por tanto, son indicadores que no deben conducir mecánicamente a la idea de causalidad, sino ser complemento de una discusión teórica más global, comprendiendo el fenómeno en su complejidad multivariada y multidimensional.

De hecho, cabe recordar que en los fenómenos sociales existen, por lo general, variables no observadas, relaciones cruzadas no completamente medibles, y que también en este caso puede ocurrir que las variables independientes tiendan a interactuar (más allá desde lo estadístico, pues las mediciones de colinealidad no lo acusan directamente). Tal como analizamos en la revisión de la literatura, por ejemplo, las escuelas que tienen más demanda, en general, tienen también la capacidad (y el estímulo) para seleccionar a sus estudiantes o, incluso, para ser excluyentes con cierto tipo de perfil de estudiante menos aventajado. Por tanto, esto hace que este tipo de escuelas tengan menores niveles de IVE y eso se relaciona con tener puntajes SIMCE más altos. Este fenómeno se visualiza al ver el modelo óptimo de regresión, en comparación con los demás indicadores por separado. 
Finalmente, este estudio busca ser introductorio para poder ir profundizando en este campo de utilización del SAE como forma de insumo para la planificación de la oferta educativa. Los supuestos tras la presente investigación corresponden a observar qué variables puedan predecir la cantidad de demanda que tiene una escuela y así dar pie a nuevas exploraciones o profundización de las mismas:

- El estudio es una primera aproximación, muy global y gruesa, respecto de la potencialidad del SAE para sistematizar datos relacionados con la demanda y comprender, con mayor detención, la voluntad de los apoderados de diferenciar entre establecimientos cerca del domicilio en relación con otras variables.

- Intenta visualizar, con mayor precisión, espacios geográficos (comunas o barrios específicos) con alta/baja densidad demográfica y qué especificidades se dan en dichos casos respecto a la elección de escuela.

- Lo geográfico depende de otras variables: la distancia de la escuela se mide de forma lineal, no se considera el transporte público y elementos asociados. El estudio combina ciudades muy diferentes entre sí y, por tanto, hay que tomar en cuenta que, si bien en este estudio la distancia o la cantidad de postulantes cerca del establecimiento influye poco, hay que tomar esa dimensión y compatibilizarla junto con otros aspectos para un análisis más detenido.

- Es necesario abarcar el proceso SAE 2020 desde las postulaciones a otros cursos (por ejemplo, las postulaciones a primero básico, primer año de secundaria) y evaluar si las variables predictoras son iguales o diferentes a las de esta investigación.

- Además, profundizar metodológicamente, ampliando el espectro de la demanda de cada apoderado a su conjunto de preferencias. Analizar la conducta más allá de las primeras preferencias, de tal manera de comprender en ello las expectativas o qué tipo de escuelas son más rankeadas como "segundas o terceras preferencias". O bien, qué tipos de escuelas compiten entre sí, o intentando caracterizar tipos de apoderados con tipos de escuela. 
- El SAE genera un entorno favorable para comprender la sobreoferta de escuelas o la sobredemanda de postulantes en distintas zonas de Chile. De este modo se puede obtener una estimación de la "capacidad ociosa" de los establecimientos que no son demandados por las familias y analizar las escuelas menos preferidas y por qué.

Como se mencionó, otro de los objetivos de este estudio fue promover el análisis de las preferencias de los apoderados desde una perspectiva de los propios sostenedores y directivas de centros educativos, comprendiendo cómo pueden beneficiarse, aprovechando el amplio abanico de datos que se abre al observar detenidamente un proceso del SAE y la información que genera. Creemos que son insumos que las escuelas pueden ir incorporando en sus procesos de mejora.

\section{Referencias}

Adones, K (2018). Análisis de la composición del nuevo alumnado del Liceo Luis Cruz Martinez de Calama tras la entrada en funcionamiento del SAE y la percepción de la comunidad entorno a este tema. (tesis para optar al grado de Magíster en Políticas Públicas). Universidad del Desarrollo, Chile.

Allen, R. (2007). Allocating pupils to their nearest secondary school: The consequences for social and ability stratification. Urban Studies, 44(4), 751-770. DOI: https://doi.org/10.1080/00420980601184737

Arteaga, F., Paredes V. \& Paredes, R. (2018). School Segregation in Chile: Residence, Co-payment, or Preferences. Documento de Trabajo, Departamento de Ingeniería Industrial, PUC.

Atria, F. (2012). La Mala Educación. Ideas que inspiran al movimiento estudiantil en Chile. Santiago de Chile: Catalonia.

Bellei, C. (2015). El gran experimento. Mercado y privatización de la educación chilena. Santiago, Chile: LOM.

Bellei, C. (2016). Dificultades y resistencias de una reforma para desmercantilizar la educación. RASE: Revista de la Asociación de Sociología de la Educación, 9(2), 232-247.

Bellei, C., Gonzalez, P. y Valenzuela, J. P. (2010). Fortalecer la educación pública: un desafío de interés nacional. En C. Bellei, D. Contreras y J. 
P. Valenzuela (Eds.), Ecos de la Revolución Pingüina. Avances, desafios y silencios de la reforma educacional. Santiago, Chile: Pehuén.

Bellei, C., Canales, M, Orellana, V. y Contreras, M. (2016). Elección de escuela en sectores populares: Estado, mercado e integración social. Revista Austral de Ciencias Sociales, (31), 95-110.

Berends, M. (2015). Sociology and School Choice: What We Know After Two Decades of Charter Schools. Annu. Rev. Sociol., (41), 159-180.

Bifulco, R., Ladd, H. F. \& Ross, S. L. (2009). Public school choice and integration evidence from Durham, North Carolina. Social Science Research, 38(1), 71-85. DOI: https://doi.org/10.1016/j. ssresearch.2008.10.001

Bolívar, A., López, J. y Murillo, F. (2014). Liderazgo en las instituciones educativas. Una revisión de líneas de investigación. Fuentes: Revista de la Facultad de Ciencias de la Educación, (14), 15-40.

Bonal, X. y Zancajo, A. (2020). Elección de escuela, movilidad y segregación escolar del alumnado vulnerable en Barcelona. REICE. Revista Iberoamericana sobre Calidad, Eficacia y Cambio en Educación, 18(4), 197-218. DOI: https://doi.org/10.15366/reice2020.18.4.008

Burgess, S., Greaves, E., Vignoles, A, \& Wilson, D. 2015. What parents want: School preferences and school choice. The Economic Journal, 125(587): 1262-1289. DOI: https:// doi.org/10.1111/ecoj.12153

Calvo, A., Haya, I. y Susinos, T. (2012). El rol del orientador en la mejora escolar. Una investigación centrada en la voz del alumnado como elemento de cambio. Revista de Investigación en Educación, 2(10), 7-20.

Canales, M., Bellei, C. y Orellana, V. (2016). ¿Por qué elegir una escuela privada subvencionada? Sectores medios emergentes y elección de escuela en un sistema de mercado. Estudios Pedagógicos, 42(3), 89-109.

Canales, M., Guajardo, F., Orellana, V., Bellei, C. y Contreras, M. (2020). Fin del copago y nuevo Sistema de Admisión Escolar: duelo de estrato. Estudios Pedagógicos, 46(2), 299-319. DOI: https://dx.doi.org/10.4067/ S0718-07052020000200299

Carrasco, A., Oyarzún, J., Bonilla, A., Honey, N. y Díaz, B. (2019). La experiencia de las familias con el Nuevo Sistema de Admisión Escolar: Un cambio cultural en marcha. Serie Estudios en Justicia Educacional, 2.

Carrasco, A. y Honey, N. (2019). Nuevo Sistema de Admisión Escolar y su capacidad de atenuar la desigualdad de acceso a colegios de calidad: al inicio de un largo camino. Estudios en Justicia Educacional, 1. 
Cea D’Ancona, Ma. A. (2004) Análisis multivariable. Teoría y práctica en la investigación social. Madrid: Síntesis.

Contreras, D., Hojman, D., Huneeus, F. y Landerretche, O. (2011). El lucro en educación escolar. Evidencia y desafíos regulatorios. Trabajos de Investigación en Políticas Públicas, No 10. Santiago, Chile: Departamento de Economía, Universidad de Chile.

Córdoba, C. (2014). La elección de escuela en sectores pobres: resultados de un estudio cualitativo. Psicoperspectivas, Viña del Mar: Pontificia Universidad Católica de Valparaíso, 13(1), 56-67.

Córdoba, C., Farris, M. \& Rojas, K. (2017). Discussing school socioeconomic segregation in territorial terms: the differentiated influence of urban fragmentation and daily mobility. Investigaciones Geográficas, (92), 4350.

Corvalán, J. y Román, M. (2016), Dicen que esta escuela es mala, pero nosotros la encontramos buena. Elección de escuela en familias pobres en Chile. En J. Corvalán, A. Carrasco y J. E. García Huidobro, Mercado escolar y oportunidad educacional. Libertad, diversidad y desigualdad (pp. 209-231). Santiago, Chile: CEPPE, Ediciones UC.

Duk, Cynthia y Murillo, F. Javier. (2019). Segregación Escolar y Meritocracia. Revista latinoamericana de educación inclusiva, 13(1), 1113. DOI: https://dx.doi.org/10.4067/S0718-73782019000100011

Elacqua, G. (2012). The impact of school choice and public policy on segregation: Evidence from Chile. International Journal of Educational Development, 32(3), 444-453. DOI: https://doi.org/10.1016/j.ijedudev 2011.08 .003

Espinosa, L. (2017). Entre la autonomía y la inclusión. Análisis de la implementación del sistema de admision escolar centralizado en la comuna de Providencia 2013-2015 (tesis para optar al grado de magíster en Gestión de Políticas Públicas). Universidad de Chile, Santiago, Chile.

Eyzaguirre, S., Hernando, A. y Blanco, N. (2018). Cargando con la mochila ajena. Resultados y desafíos del nuevo Sistema de Admisión Escolar. Puntos de Referencia, 498. Centro de Estudios Públicos.

Flores, C. y Carrasco A. (2013). (Des)igualdad de oportunidades para elegir escuela: Preferencias, libertad de elección y segregación escolar. Santiago, Chile: Espacio Público. Documento de Referencia.

Fukushi, K. (2010). El nuevo alumno y el desafío de la meritocracia: Análisis del cambio cultural en la educación superior chilena. Calidad en la Educación, (33). 
González, M. (2011). Dirección y Liderazgo educativo en los centros escolares. En M. González (Coord.). Innovaciones en el gobierno y gestión de los centros escolares (pp. 163-183). Madrid: Síntesis.

Hargreaves, A. \& Shirley, D. (2012). The global fourth way. Thousand Oaks, CA: Corwin.

Hernández, M. y Raczynski, D. (2015), Elección de escuela en Chile: De las dinámicas de distinción y exclusión a la segregación socioeconómica del sistema escolar. Estudios Pedagógicos, XLI(2), 127-141.

Kye, S. H. (2018). The persistence of white flight in middle-class suburbia. Social Science Research, (72), 38-52. DOI: https://doi.org/10.1016/j. ssresearch.2018.02.005

Landerretche, O. y Lillo, N. (2011). Percepciones sobre movilidad social y meritocracia: Un estudio para Chile usando la Encuesta de Trabajo y Equidad. Working Papers, wp331. Universidad de Chile, Departamento de Economía.

Ley 20.845. (2015). De Inclusión Escolar que regula la admisión de los y las estudiantes, elimina el financiamiento compartido y prohíbe el lucro en establecimientos educacionales que reciben aportes del Estado del Ministerio de Educación. Diario Oficial de la República de Chile. Recuperado de: https://www.leychile.cl/ Navegar?idNorma=1078172

Musset, P. (2012). School choice and equity: Current policies in OECD countries and literature review. OECD Education Working Papers, (66), $1-51$.

OECD. (2014). The importance on school leadership. In TALIS 2013 Results: An International Perspective on Teaching and Learning. Paris: OECD.

OECD. (2019). Balancing school choice and equity: An international perspective based on PISA. OECD Publishing. DOI: https://doi. org/10.1787/2592c974-en

Orellana, V., Caviedes, S., Bellei, C. y Contreras, M. (2018). La elección de escuela como fenómeno sociológico. Una revisión de literatura. Revista Brasileira de Educação, (23), e230007. Epub February 01, 2018. DOI: https://dx.doi.org/10.1590/s1413-24782018230007

Otero, G., Carranza, R. \& Contreras, D. 2017. 'Neighbour- hood effects' on children's educational achievement in Chile: The effects of inequality and polarization. Environment and Planning A, 49(11), 2595-2618. DOI: https:// doi.org/10.1177/0308518X17731780

Peña, M. y Toledo, C. (2017) Discursos sobre clase social y meritocracia de escolares vulnerables en Chile. Cadernos de Pesquisa, 47(164). 
Programa de las Naciones Unidas para el Desarrollo. (2017). Desiguales: orígenes, cambios y desafíos de la brecha social en Chile. Santiago, Chile: Programa de las Naciones Unidas para el Desarrollo.

Rodríguez, P., Valenzuela, J. P., Suchan, K., Truffello, R., Norel, N., Allende, C., Mondaca, J. y Céspedes, J. (2016). Determinando el acceso real de los estudiantes a establecimientos educacionales efectivos para generar politicas públicas que mejoren la provisión de educación de calidad. Documento de Trabajo N¹9. Centro de Investigación Avanzada en Educación (CIAE). Recuperado de: http:// www.ciae.uchile.cl/index.php?page=view_ publicaciones\&id_publicaciones=637\&langSite $=$ es

Rodríguez, C., Espinosa, D. y Padilla, G. (2020). Dónde quiero que estudien mis hijos/as: caracterización de la oferta educativa y sus niveles de demanda en Chile. Revista de estudios y experiencias en educación, 19(41), 57-70. DOI: https://dx.doi.org/10.21703/rexe.20201941rodriguez4

Santos, H. y Elacqua, G. (2016). Segregación socioeconómica escolar en Chile: Elección de la escuela por los padres y un análisis contrafactual teórico. Revista CEPAL, (119):133-148.

Sancerni, M. y Lis, A. (2013). A propósito de la innovación educativa. EDETANIA. Estudios y propuestas socio-educativas, (44), 209-218.

Sillard, M., Garay, M. y Troncoso, I. (2018). Análisis al Nuevo Sistema de Admisión Escolar en Chile: La Región de Magallanes como experiencia piloto. Calidad en la Educación, (49), 112-136.

Scheilcher, A. (2012). Preparing Teachers and Developing School Leaders for the 21st Century: Lessons from Around the World. Paris: OECD.

Treviño, E. (2018). Diagnóstico del Sistema Escolar: las reformas educativas 2014-2017. En I. Sánchez (Ed.), Ideas en Educación: reflexiones y propuestas desde la UC. Santiago, Chile: Ediciones UC.

Treviño, E., Valenzuela, J. P., Villalobos, C., Béjares, C., Wyman, I. y Allende, C. (2018). Agrupamiento por habilidad académica en el sistema escolar. Nueva evidencia para comprender las desigualdades del sistema educativo chileno. Revista mexicana de investigación educativa, 23(76), 45-71. Recuperado en 06 de agosto de 2020, de http://www.scielo.org.mx/scielo.php?script=sci_arttext\&pid=S140566662018000100045\&lng=es\&tlng=es.

Valenzuela, J. M., Labarrera, P. y Rodríguez, P. (2008). Educación en Chile: Entre la continuidad y las rupturas. Principales hitos de las políticas educativas. Revista Iberoamericana de Educación, (48), 129-145.

Valenzuela, J., Bellei, C. \& Ríos, D. (2014). Socioeconomic school segregation in a market-oriented educational system. The case of Chile. Journal of 
210 LOS ATRIBUTOS DE LOS ESTABLECIMIENTOS EDUCACIONALES QUE PUEDEN PREDECIR LA PREFERENCIA POR PARTE DE LOS APODERADOS - M. Garay, M. Sillard

Education Policy, 29(2), 217-241. DOI: https://doi.org/10.1080/0268 0939.2013.806995

Villalobos, C. y Quaresma, M. L. (2015). Sistema escolar chileno: características y consecuencias de un modelo orientado al mercado. Convergencia, 22(69), 63-84.

Weinstein, J. \& Muñoz, G. (2014). What do we know about school principals? Lessons from the case of Chile. Interamerican dialogue. Recuperado de: https://prealblog.files.wordpress.com/2014/12/final-school-principalsin-chile-english-fs-12-1-14.pdf

Recibido:19/09/2020

Aceptado: 03/05/2021 


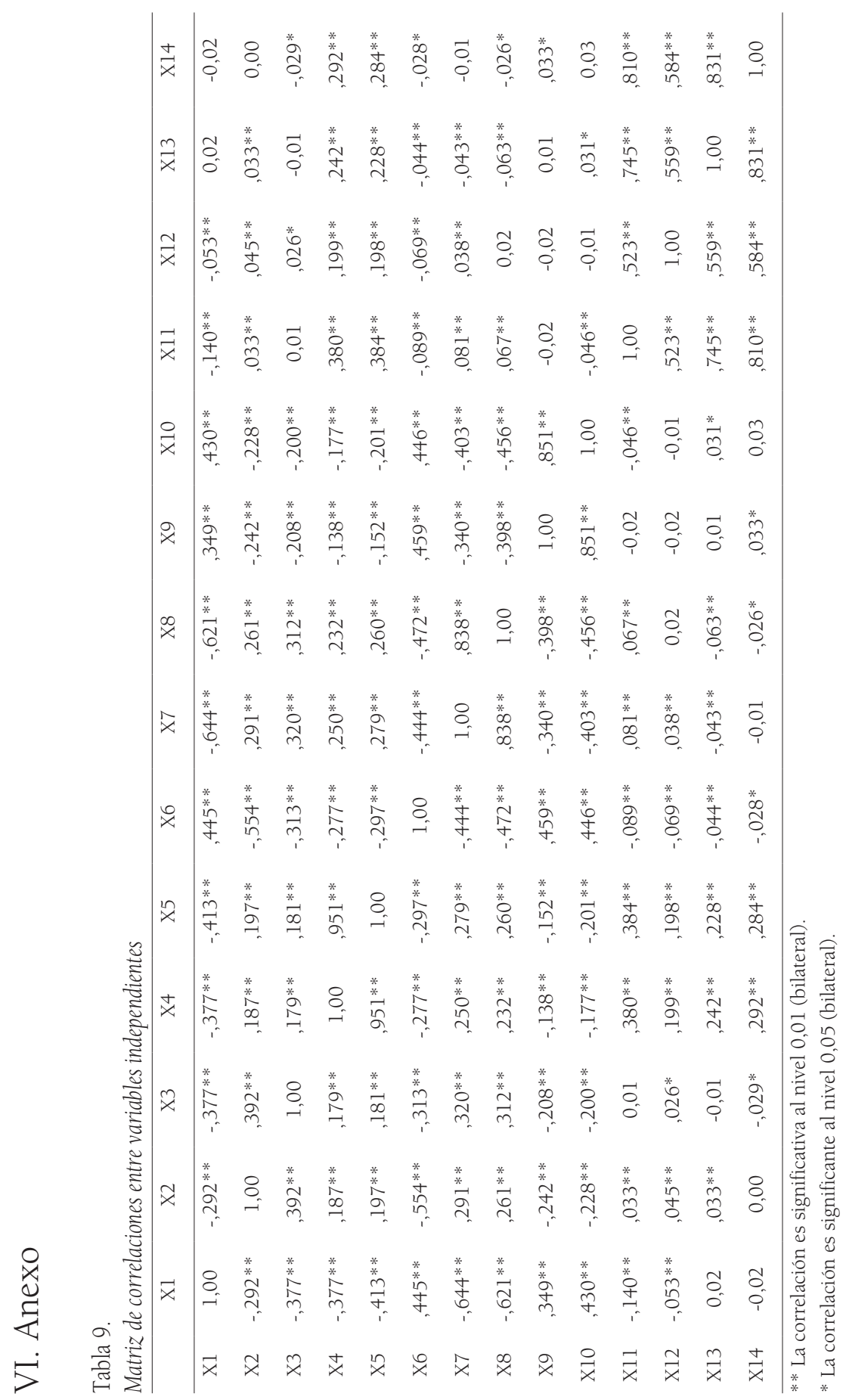

